\title{
E-textiles para la formación de profesorado en las áreas STEM
}

\section{Paola Guimeráns Sánchez}

Doctora por la Universidad Complutense de Madrid, España

pguimerans@gmail.com

\begin{abstract}
Resumen
En este artículo se aborda la formación del profesorado de educación primaria y secundaria con el objetivo de capacitarlo para el diseño y la aplicación de propuestas didácticas innovadoras enmarcadas en el enfoque STEAM. Se valora la importancia que posee el área multidisciplinar de los e-textiles en la educación y se argumenta cómo el kit de construcción LilyPad puede conformar una pieza clave para que funcionen al mismo nivel todas estas materias en el aula. En este trabajo se describen dos talleres teórico-prácticos, que cuentan con la participación de un total de 32 profesores expertos en diferentes especialidades. Estas acciones educativas se incluyen en el marco de una colaboración entre la autora del texto y los organizadores de proyectos de innovación educativa Aula 3i, que promueven las áreas de Innovación y Nuevas Tecnologías del Cabildo de Fuerteventura y el Parque Tecnológico de Fuerteventura.
\end{abstract}

Palabras clave: STEM, e-textiles, enseñaza de la programación, pensamiento computacional, innovación educativa

\begin{abstract}
This article deals with the training of primary and secondary education teachers with the aim of qualify them for the design and application of innovative didactic proposals framed in the STEAM approach. The importance of the multidisciplinary area of e-textiles in education is assessed and it is discussed how the LilyPad construction kit can be a key element so that all these subjects works in the same level in the classroom. This paper describes two theoretical-practical workshops that have the participation of a total of 32 expert teachers in different specialties. These educational actions are included within the framework of a collaboration between the author of the text and the organizers of educational innovation projects of the Aula3i project, which promotes the areas of Innovation and New Technologies of the Fuerteventura Cabildo and the Fuerteventura Technology Park.
\end{abstract}


Keywords: STEM, e-textiles, programming, computational thinking, innovation in education

\section{Introducción}

La integración del pensamiento computacional en el aprendizaje informal y formal supone una tendencia creciente. De hecho, desde que en el año 2006 Jeannette Wing (2006) publicara el influyente artículo «Computational thinking», no han dejado de surgir en diferentes países propuestas educativas con el objetivo de incorporar esta nueva alfabetización digital en el currículo escolar; en especial, por la importancia y potencia de esta habilidad para educar a una nueva generación de individuos con una comprensión mucho más profunda del mundo. Al respecto, bajo la experimentación libre que caracteriza a los procesos maker (Kalil 2010; Martinez \& Stager, 2013; Peppler et al. 2016), destacamos el surgimiento, en Estados Unidos, de un nuevo modelo de educación que responde a las siglas de STEAM (Maeda, 2012). Con este enfoque se busca, favoreciendo la teorización e integración del arte, fortalecer la enseñanza de la ciencia, la tecnología, la ingeniería y las matemáticas, aglutinadas comúnmente bajo las siglas en inglés STEM (Sanders, 2009). Como parte de las iniciativas educativas englobadas bajo esta denominación, autores como Peppler (2016) señalan el floreciente campo de los e-textiles y sus aplicaciones a la educación, como pieza clave para fomentar el pensamiento computacional, y que funcionen al mismo nivel todas estas materias.

En este texto detallamos dos talleres de iniciación al campo de los e-textiles orientados al profesorado de educación primaria y secundaria. La autora de este artículo, e impulsora de la iniciativa educativa Aula STEAM, diseña e imparte esta formación como parte de una colaboración con los organizadores de proyectos de innovación educativa Aula 3i. En las conclusiones de este texto, se reportan los principales resultados de las experiencias obtenidas en dichos talleres.

\section{La importancia de los e-textiles en la educación}

Tradicionalmente, los profesores de Tecnología han utilizado en el aula la placa de prueba, los cables y el soldador para enseñar a sus alumnos conceptos sobre electricidad y circuitos. Ahora bien, de un tiempo a esta parte, los e-textiles se han posicionado como un modelo alternativo que está resultando ser más eficaz. Esto sucede después de que varios autores hayan observado que, en contextos de educación formal o vinculados a la cultura maker, el desarrollo de proyectos de e-textiles promueve el interés de los estudiantes por la 
informática. De igual forma, con este enfoque se favorece la retención de conceptos complejos y abstractos, así como la electrónica resulta mucho más accesible (Tofel-Grehl et al., 2017). En términos generales, los e-textiles, también conocidos como «textiles electrónicos», son dispositivos electrónicos o wearables que, en español, se traduciría como «tecnología vestible, usable o llevable». En palabras de Peppler (2017), "cuando nos referimos a los e-textiles en la educación, nos referimos a algo tan sencillo como hacer funcionar un circuito eléctrico con un solo led y hacer que este brille dentro de una pulsera, o a otro tipo de proyectos más avanzados que requiere de programación e involucra múltiples sensores, sonido, luces y movimiento".

Desde un punto de vista investigativo, el área de estudio de los e-textiles se sitúa en la intersección de la moda, el diseño industrial, el de muebles, el de interacción, la ciencia computacional y el arte de los nuevos medios, entre otros. En este campo multidisciplinario se utilizan novedosos materiales flexibles, conductores e inteligentes, con los cuales se puede conseguir resultados muy visuales y estéticos (Orth, et al., 1998). Ahora bien, hasta hace poco tiempo, este campo de estudio era complejo e inaccesible y se situaba en torno a laboratorios o centros de investigación. Sin embargo, tras el cambio de milenio, gracias al auge de la cultura maker, la filosofía DIY y la web 2.0, el campo de los e-textiles se democratiza, de modo que se ha vuelto más accesible para todos los públicos; especialmente, tras la aparición del kit de construcción educativo LilyPad, cuya placa LilyPad Arduino, como su nombre indica es compatible con Arduino. De hecho, esta nueva herramienta digital cosible representará para el campo de la tecnología aplicada a la educación una gran oportunidad, pues su placa ha sido ideada y diseñada para ampliar la participación e incluir a minorías en los campos STEM (Buechley \& Hill, 2010). Este kit, pensado en torno a la corriente pedagógica del construccionismo, resultará ser altamente efectivo en el entorno educativo para el fomento del pensamiento computacional y para que personas sin experiencia previa puedan diseñar y construir sus propios wearables y otros artefactos textiles (Buechley, 2010).

Otro hecho destacable reside en que el componente artístico de los e-textiles propondrá un enfoque particularmente atractivo y accesible para que nuevas y diversas audiencias se acerquen a las áreas científico-tecnológicas (Qiu et al., 2013). Por otra parte, Kafai y otros autores (Kafai et al., 214), en sus investigaciones, habrían comprobado que, desarrollando proyectos de e-textiles, se refuerza en el alumnado una identidad maker y se cambia sus ideas sobre quiénes pueden participar en las áreas técnicas. 


\section{E-textiles como un camino para potenciar la formación en las áreas STEM}

Siendo conscientes de la responsabilidad y necesidad de formación continua que tiene los docentes para ser innovadores en el aula, a continuación describimos dos talleres dirigidos a profesores de educación primaria y secundaria. Tales talleres tienen como objetivo capacitarlos para el diseño de propuestas didácticas vinculadas al campo de los e-textiles. El fin de estos talleres es ofrecer una formación inicial a la electrónica y programación para que puedan integrar este tipo de habilidades en su práctica docente y desarrollar actividades que les permitan fomentar el pensamiento computacional a su alumnado. Asimismo, se pretende que se familiaricen con las nuevas metodologías, lo cual podría aplicarse, a su vez, a varias áreas.

\subsection{Taller iniciación a los E-textiles. II Encuentro de Profesores Maker}

Este taller teórico-práctico de iniciación a los e-textiles se imparte como una actividad para docentes en el marco del II Encuentro de Profesores Maker celebrado en octubre de 2016 en el Parque Tecnológico de Fuerteventura. El objetivo de este encuentro es dar a conocer propuestas innovativas a los docentes de la isla para que estos impulsen en el aula el fomento de las vocaciones científico-tecnológicas, así como promover el movimiento maker y la importancia que tiene en el campo educativo. El taller tuvo una duración de ocho horas y sirvió de iniciación básica a la electrónica y exploración de nuevas técnicas y materiales para la integración de la tecnología en textiles. Esta iniciativa pretende familiarizar a los docentes con este campo emergente y mostrarles varias iniciativas y recursos online, así como explicarles la importancia de los makerspaces y cómo diseñar estos espacios de trabajo colaborativo en la escuela. Al taller asistieron 22 profesores, de los cuales 16 mujeres y 6 hombres fueron de las siguientes áreas: Tecnología, Informática y Educación Plástica y Visual. Los profesores impartían clase en cinco IES (institutos de enseñanza secundaria), un CEO (centro de enseñanza obligatoria), cinco CEIP (centro de educación infantil y primaria), un CIFP (centro integrado de formación profesional) y también un CEPA (centro público de educación de personas adultas). De los 22 participantes, 9 no tenían ningún conocimiento de electrónica; entre este grupo, se hallaban todos los profesores de educación primaria.

El taller comienza con una demostración de cómo coser un circuito básico eléctrico en una pieza textil utilizando un hilo conductor de electricidad. A continuación, se realiza una breve introducción acerca de cómo crear circuitos en paralelo y en serie, un estándar que se incluye en el contenido curricular de primero de la ESO; también se explica cómo construir un interruptor utilizando textiles. En este momento, se invita a los asistentes a que ellos mismos construyan su propio circuito (Figura 1). Para ello, se les entrega un kit con todos los materiales necesarios y una hoja de instrucciones para desarrollar la actividad. Luego, se 
muestran diferentes proyectos de e-textiles realizados por profesores en equipo, y colaborativamente, en varios makerspaces. En ese instante, se invita a los asistentes a que, inspirados por lo que han aprendido hasta el momento, diseñen su propio prototipo utilizando las gorras o camisetas que se encuentran sobre la mesa (Figura 2). La sesión termina con la presentación de los proyectos y se discute acerca de diferentes aplicaciones en el aula.
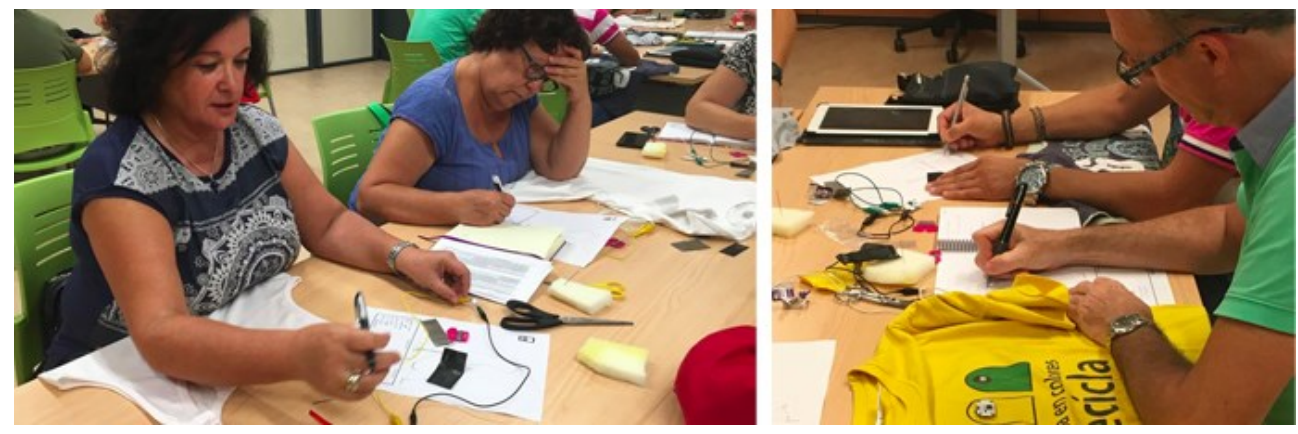

Fig. 1 y 2.. Profesores contruyendo un circuito y comenzado a diseñar su proyecto.

\subsection{LilyPad para profesoras. Día Internacional de la Mujer y Niña Científica}

Este taller teórico-práctico se imparte en febrero de 2018 como parte de las acciones realizadas durante el Día Internacional de la Mujer y Niña en la Ciencia, un evento impulsado por la Agencia Canaria de Investigación, Innovación y Sociedad de la Información (ACIISI) y el Cabildo y el Parque Tecnológico de Fuerteventura. El objetivo de este taller es destacar el papel relevante de mujeres pioneras en sectores estratégicos de la $\mathrm{I}+\mathrm{D}+\mathrm{I}$; en esta ocasión, la labor de la científica y diseñadora Leah Buechley como creadora del kit de construcción educativo LilyPad. El taller se enfocó solo a profesoras, con el fin de crear debate entre ellas y analizar, en conjunto, cómo potenciar las vocaciones científico-tecnológicas; en especial, entre las alumnas. En todo caso, se realizó para cuestionarse si el cambio en las herramientas utilizadas en el aula podría resultar en una mejora en los porcentajes de alumnas que deciden seguir los itinerarios tecnológicos. El taller tuvo una duración de ocho horas y participaron 10 profesoras: seis de Tecnología, dos de Educación Primaria, una de Física y Química y una de EPV (Educación Plástica y Visual).

El taller comienza con una introducción al trabajo de Leah Buechley y se muestran varios proyectos educativos realizados por sus estudiantes entre 2009 y 2014 cuando ella dirigía el grupo High-Low Tech Media Lab del MIT, Instituto Tecnológico de Massachusetts. A continuación, se ofrece a los asistentes un kit y se les explica qué es un microcontrolador. En este momento, se les indica que la placa LilyPad contiene el mismo microprocesador 
que un Arduino estándar y que se programa usando el mismo software de código y diseño abierto. Además, se explica que el kit consta de un conjunto de piezas de entrada y salida que se pueden controlar, como sensores de temperatura o sensores de luz. Por último, se les comenta que, para programar estos componentes aunque existen otras parecidas, se utilizará una plataforma de programación visual llamada Mblock y cómo funciona. A partir de aquí, se divide a las asistentes en dos grupos.

En un primero, se juntan a las profesoras de educación primaria y a las de educación secundaria que utilizan la programación y la robótica, y que ya han asistido al taller anterior. A este grupo se le entrega directamente un kit con todos los materiales necesarios para diseñar un bolso textil interactivo (Figura 3) y se le facilita información y recursos online, como actividades y ejercicios ya terminados. En un segundo grupo, se reúne al resto de las docentes que, o bien no tienen conocimientos previos de electrónica, o bien no conocen la existencia de nuevos materiales como el hilo o el textil conductor de electricidad. Tras una introducción, se los anima a desarrollar su primer circuito en textil (Figura 4). Para ello, se les entrega un kit con todos los materiales necesarios para realizar la actividad. Al terminar, igual que a sus compañeras, se les brindan las instrucciones necesarias para que diseñen, en este caso en equipo, un bolso textil interactivo. La sesión termina con la presentación de los proyectos y se discute sobre diferentes aplicaciones en el aula.
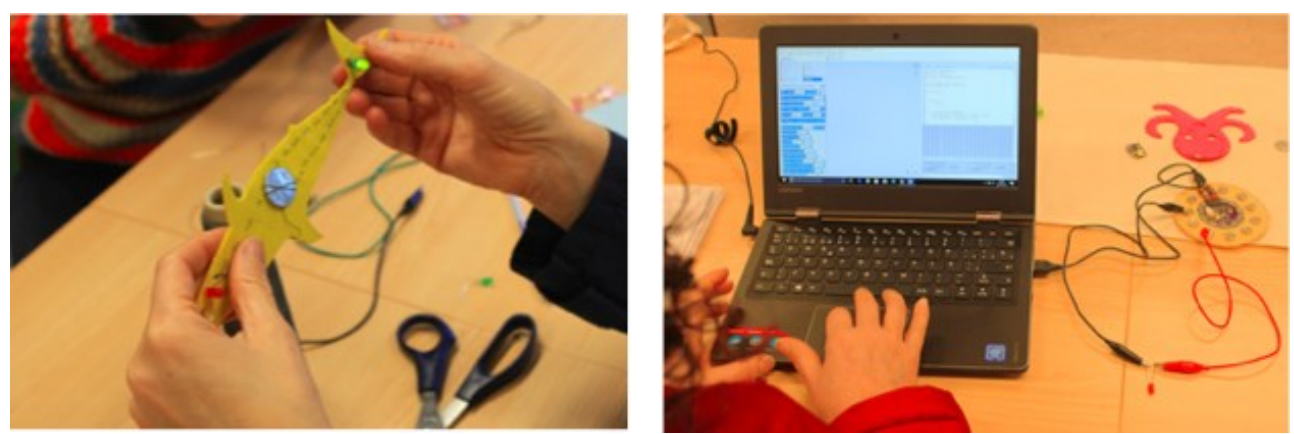

Fig. 3 y 4. Profesoras creando su primer circuito en textil y programando con Mblock la placa Lilypad Arduino

\section{Resultados}

De los docentes que, en 2016, asistieron al primer taller y que actualmente continúan trabajando, destacamos que el profesorado de tres CEIP de primaria ha profundizado y avanzado en la introducción del pensamiento computacional en el aula. De hecho, una profesora ha creado un makerspace llamado Aula STEAM. Los resultados de las 
evaluaciones muestran que el hecho de conocer nuevos materiales, como el hilo o el textil conductor de electricidad, ha llevado a varios profesores de educación primaria a querer explorar y experimentar en el aula con herramientas que les permiten conectar el mundo físico con el digital; por ejemplo, se han realizado diferentes actividades en el aula utilizando la placa de código abierto Makey Makey y experimentado con estos nuevos materiales. Por último, se ha de indicar que, en los resultados de las encuestas, la mayoría de los profesores coinciden en que, aunque tienen interés en profundizar en las posibilidades que representa apostar por el enfoque maker en educación, la falta de formación, de materiales y que no existan unidades didácticas preparadas de forma sencilla les supone un gran impedimento para poder avanzar. Ahora bien, esta situación se ha ido solucionando en este último año con la compra de materiales y la creación en la isla de una teknoteca, un sistema de préstamo implantado desde el proyecto Aula $3 \mathrm{i}$.

De las profesoras que asistieron en 2018 al segundo taller, y tras hacer una valoración general de sus opiniones, concluimos que el kit de LilyPad y la plataforma de programación visual llamada Mblock les han parecido una sencilla y valiosa herramienta de apoyo al proceso de enseñanza-aprendizaje. Entre las iniciativas que se han realizado para avanzar en la introducción de la programación y el pensamiento computacional en el aula, indicamos que, en un centro de primaria, se realizó una actividad demostrativa con alumnos utilizando el kit de LilyPad y el entorno de bloques Mblock. Por otra parte, en tres centros de secundaria, se han realizado diferentes actividades sencillas con e-textiles como, por ejemplo, la creación de una gorra con un sensor de luz, un estandarte textil luminoso o tarjetas textiles interactivas. Destacamos, de forma importante, que una profesora de Educación Plástica y Visual ha propuesto en su escuela crear un "e-fanzine" cultural de varias temáticas con sus alumnos. Esta experiencia interdisciplinar fue llevada a cabo durante este curso en el IES Puerto del Rosario entre los departamentos de Tecnología y Educación Plástica y Visual, y tuvo como objetivo crear una fanzine interactivo integrando pequeños componentes electrónicos y nuevos materiales conductores de electricidad en el papel. Para realizar esta experiencia, se trabajó sobre el proyecto con alumnado de primero de la ESO alrededor del tema de electricidad y circuitos eléctricos. Tras analizar los resultados, ambas docentes concluyen que la experiencia ha sido muy atractiva para los estudiantes, quienes se mostraron muy sorprendidos al descubrir la existencia y propiedades expresivas de nuevos materiales, como el hilo conductor de electricidad o el adhesivo de cobre.

En particular, la profesora de Tecnología de primero de la ESO estimó que, si bien en otros cursos había observado a algunos estudiantes, en especial alumnas más retrasadas en la construcción de los circuitos cuando manipulaban los cables, esto no ha ocurrido cuando se ha propuesto el uso de estos materiales. De hecho, según los resultados que muestra la encuesta, el desarrollo de dichas prácticas ha resultado ser particularmente atractivo y 
accesible para el alumnado en general y, por ello, se repetirá la experiencia el próximo curso. Por igual, ambas profesoras coinciden en lo importante que es fomentar el modelo basado en proyectos en el aula, y en las ventajas que representa enseñar, desde las artes plásticas y visuales a los estudiantes, diferentes conceptos importantes de las áreas STEM.

Consideramos importante citar que, con el objetivo de fomentar el desarrollo de estas iniciativas transversales en la escuela y para que estas puedan ser evaluadas como parte del currículo escolar, las docentes siguieron un criterio de evaluación por asignatura. En relación con el criterio establecido para Tecnología de primero de la ESO, se valoró, en los resultados de los trabajos, si el alumno había analizado y descrito la naturaleza de la corriente eléctrica y sus efectos, así como el hecho de si había diseñado y simulado circuitos eléctricos con operadores elementales, utilizando la simbología adecuada para analizar su funcionamiento. También se estimó si, en la creación del e-fanzine, el alumno diseñó y creó un producto tecnológico sencillo de forma guiada, identificando y describiendo las etapas necesarias, además de realizar las operaciones técnicas previstas en el plan de trabajo para investigar su influencia en la sociedad. Siguiendo el criterio de evaluación de la asignatura de Educación Plástica, Visual y Audiovisual de primero de la ESO, en los trabajos finales se tuvo en cuenta si se produjo la creación de composiciones abstractas o figurativas con diferentes intenciones comunicativas; asimismo, si el alumnado aplicó diversas técnicas secas, húmedas y mixtas, utilizando distintos soportes y materiales, con el fin de construir una visión global de distintas técnicas gráfico-plásticas. Por último, cabe mencionar que, si bien el kit de Arduino estándar como herramienta educativa se está introduciendo en estos momentos en tercero y cuarto de la ESO, así como en primero de BACh (bachillerato), en las encuestas varias profesoras de Tecnología han mostrado su interés en que también se valore incluir el kit de LilyPad, ofreciendo ambas herramientas al alumnado para que puedan desarrollar sus proyectos.

\section{Discusión y conclusiones}

A la luz de esta experiencia, podemos concluir que la realización de proyectos de e-textiles representan una gran oportunidad; en particular, para fomentar tanto en el docente como en el alumno el desarrollo de nuevas habilidades y formas de pensamiento que resultan fundamentales para desenvolverse en el mundo del siglo XXI. En referencia al nivel de logros obtenidos en los talleres, y coincidiendo con los efectos reportados en otras investigaciones similares (Knobel \& Kalman, 2016), todo apunta a que iniciarse en el campo de los e-textiles y el kit de construcción LilyPad capacita a los docentes para fomentar las STEM y conceptos relacionados con la educación artística en el aula. De hecho, conecta a profesores de diferentes áreas para que colaboren en la construcción de 
investigaciones y actividades basadas en proyectos que incorporen el pensamiento computacional y la programación.

En relación con el debate sobre cómo introducir las habilidades de pensamiento computacional en el currículo escolar para formar a una generación de sujetos creativos y competentes para abordar problemas sistémicos y complejos, todo indica que el enfoque STEAM puede resultar ser más positivo que el STEM para que se produzca un mayor acercamiento de las alumnas a los itinerarios tecnológicos. Ahora bien, en España los contenidos relacionados con la programación y el pensamiento computacional, aunque son escasos, se encuentran vinculados al desarrollo de proyectos de robótica. En realidad, en el currículo de la enseñanza obligatoria que está vigente en el contexto educativo español, se valora la robótica educativa y predomina el enfoque STEM para aprender programación y desarrollar las disposiciones básicas para fomentar el pensamiento computacional (INTEF, 2018). Además, se trata, en muchas ocasiones, de asignaturas optativas, por lo que un estudiante puede cursar toda la educación obligatoria sin haber recibido apenas ningún tipo de formación en relación con estas habilidades.

No obstante, todos coincidimos con que estamos en un momento de transición en el terreno educativo. Por esto, si se propone comenzar a programar y a desarrollar el pensamiento computacional a través de la robótica, consideramos importante que se valore también el campo de los e-textiles y el uso de nuevos materiales vinculados a este campo, puesto que se presenta como otra opción igual de válida para realizar este mismo cometido. De hecho, la placa LilyPad utiliza el mismo microcontrolador y programa que el tradicional Arduino, utilizado para prototipar robots. Ahora bien, aunque de manera diferente, utilizando ambas placas, se introducen los mismos conceptos y habilidades en el aula, la única diferencia estriba en el tipo de proyectos que se desarrollan. Incluso, a diferencia de la robótica educativa tradicional, los e-textiles aplicados a la educación permiten desarrollar la expresión y la creatividad por medio del arte, y ofrecen la apertura a nuevos materiales, propuestos como un elemento motivador e interesante para el profesor y el alumnado.

Por último, se debe señalar que este estudio y sus resultados se muestran como una primera aproximación y reflexión a la integración efectiva de tal enfoque en el contexto escolar. Somos conscientes de que muchos de los materiales vinculados al campo de los e-textiles son relativamente nuevos y de que no existe una gran variedad de recursos en español. Esto sucede porque la mayoría de los estudios sobre e-textiles y educación se han desarrollado en Estados Unidos. Con este documento, estimamos propagar y ampliar el acceso a este campo; en especial, animados por los resultados obtenidos en Ontario (Hughes \& Morrison, 2018) que reflejan las ventajas que este campo representa para fomentar la creatividad y el interés de las adolescentes y niñas por las disciplinas STEM. 


\section{Agradecimientos}

Muchas gracias a todas las personas e instituciones que han hecho posible la realización de este estudio, en especial, al Cabildo de Fuerteventura, a Manuel Miranda, a Marta Cabrera y a Rayco León, a María Inmaculada Jordán Pérez y, a todos los docentes participantes.

\section{Referencias}

Buechley, L. (2010).LilyPad Arduino: rethinking the materials and cultures of educational technology. Proceedings of the $9^{\text {th }}$ International Conference of Learning Science.

Buechley, L. \& Hill, B. (2010). LilyPad in the wild: how hardware's long tail is supporting new engineering and design communities. Proceedings of the Designing Interactive Systems.

Hughes, J. \& Morrison, L. (2018). The use of E-textiles in Ontario Education. Canadian Journal of Education. Revue canadienne de l'éducation 41:1.

INTEF (2018). Informe "Programación, robótica y pensamiento computacional en el aula". Situación en España, Enero de 2018. Ministerio de Educación, Cultura y Deporte. En : https://www.mecd.gob.es/prensa-mecd/actualidad/2018/02/20180220- robot.html

Kafai, Y. B., Fields, D. A., \& Searle, K. A. (2014). Electronic textiles as disruptive designs:

Supporting and challenging maker activities in schools. Harvard Educational Review.

Kalil, T (2010). Remarks on innovation, education, and the Maker Movement. Retrieved Febrero 14, 2018, en http://radar.oreilly.com/2010/10/innovation-education-and-the-m.html

Knobel, M., Kalman J., (2016) New Literacies and Teacher Learning: Professional Development and the Digital Turn. Peter Lang.

Maeda, J. (2012). STEM to STEAM: Art in K-12 is Key to Economy, Edutopia. What Works in Education, The George Lucas Educational Fundation.

Martinez, S., \& Stager G. (2013). Invent to Learn : Making, Tinkering and Engineering in the Classroom. New Hampshire : Constructing Modern Knowledge Press.

Orth, M, Post, R. \& Cooper, E., Fabric Computing Interfaces (1998). The Proceedings of Conference on Human Factors in Computing Systems, (CHI '98), Los Angeles. 
Peppler, K., Kafai, Y., \& Halverson, E. (2016) Makeology in K-12, Higher, and Informal Education, New York, NY: Routeledge.

Peppler, K. (2016). A review of e-textiles in education and society. In Handbook of research on the societal impact of digital media: Ch.11 (pp. 219-241). Hershey, PA.

Peppler, K. (2017). The SAGE Encyclopedia of Out-of-School Learning. Sage Publications.

Qiu, K., Buechley, L., Baafi, E. \& Dubow, E. (2013). A curriculum for teaching computer science through computational textiles. In Proceedings of the 12th International Conference on Interaction Design and Children (IDC '13). ACM, New York, NY, USA, 20-27.

Sanders, M. (2009). STEM, STEM education, STEMmania. The Technology Teacher, 69(4), 20-26.

Tofel-Grehl,C., Fields, D., Searle, K., Maahs-Fladung, C., Feldon, D, Gu, G. \& Sun, C.(2017). Electrifying Engagement in Middle School Science Class: Improving Student Interest Through Etextiles pp. 406-417(12).

Wing, J. M. (2006). Computational thinking. Communications of the ACM. 49 (3):33-35. 